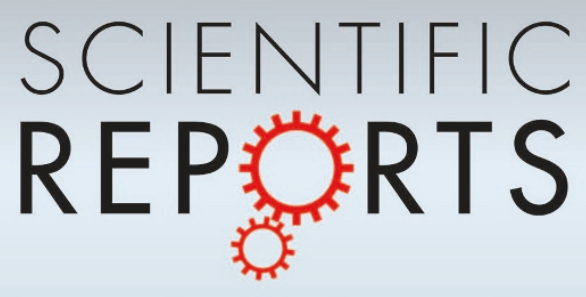

OPEN

SUBJECT AREAS:

NECROPTOSIS

TARGETED THERAPIES

COMPUTATIONAL BIOPHYSICS

SOLID-STATE LASERS

Received

30 April 2013

Accepted

3 January 2014

Published

24 January 2014

Correspondence and requests for materials should be addressed to

M.O.S. (martin.

steinhauser@emi.

fraunhofer.de)

* Current address:

Department of

Pathology, Division of

Neuropathology, Johns

Hopkins Medical

Institutions, 720 Rutland

Avenue, Baltimore, MD

21205, USA.

$\uparrow$ Current address:

Functional Neurosurgery

and Stereotaxis,

University Medical

Center Düsseldorf,

Moorenstrasse 5,

40225 Düsseldorf,

Germany.

\$ Current address:

Department of

Stereotactic

Neurosurgery,

Neurosurgical Clinic,

University Medical

Center Erlangen,

Schwabachanlage 6,

91054 Erlangen,

Germany.

\section{Characterization of a Setup to test the Impact of High-Amplitude Pressure Waves on Living Cells}

\author{
Mischa Schmidt 1,2, Ulf Kahlert²*, Johanna Wessolleck², Donata Maciaczyk², Benjamin Merkt ${ }^{3}$, \\ Jaroslaw Maciaczyk ${ }^{2} \dagger$, Jens Osterholz ${ }^{3}$, Guido Nikkhah ${ }^{2}+\&$ Martin O. Steinhauser ${ }^{1,4}$
}

\begin{abstract}
'Research Group "Shock Waves in Soft Biological Matter", Fraunhofer Institute for High-Speed Dynamics, Ernst-Mach-Institut, EMI, Eckerstrasse 4, 79104 Freiburg, Germany, ${ }^{2}$ Department of Stereotactic and Functional Neurosurgery, Neurocenter, University Medical Center Freiburg, Breisacher Straße 64, 79106 Freiburg, Germany, ${ }^{3}$ Laser Technology Group, Fraunhofer Institute for High-Speed Dynamics, Ernst-Mach-Institut, EMI, Eckerstrasse 4, 79104 Freiburg, Germany, ${ }^{4}$ Department of Chemistry, Faculty of Science, Universität Basel, Klingelbergstrasse 80, CH-4056 Basel, Switzerland.
\end{abstract}

The impact of pressure waves on cells may provide several possible applications in biology and medicine including the direct killing of tumors, drug delivery or gene transfection. In this study we characterize the physical properties of mechanical pressure waves generated by a nanosecond laser pulse in a setup with well-defined cell culture conditions. To systematically characterize the system on the relevant length and time scales (micrometers and nanoseconds) we use photon Doppler velocimetry (PDV) and obtain velocity profiles of the cell culture vessel at the passage of the pressure wave. These profiles serve as input for numerical pressure wave simulations that help to further quantify the pressure conditions on the cellular length scale. On the biological level we demonstrate killing of glioblastoma cells and quantify experimentally the pressure threshold for cell destruction.

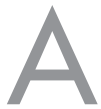

part from diagnostic ultrasound there are two different major treatment methods in clinical practice that make use of the effect of pressure waves: Extracorporeal shock wave lithotripsy (ESWL) for the treatment of urinary stone disease $e^{1,2}$ and high intensity focused ultrasound (HIFU) as a tumor treatment ${ }^{3}$. Both techniques are noninvasive without the need for open surgery because pressure pulses are generated outside the body and focused at the location of the treatment where the full destructive effect occurs ${ }^{2,4}$. Since its first application to a patient in the 1980s, ESWL is the only noninvasive method to remove urinary calculi such as kidney stones and has become the most well established treatment in this field ${ }^{1,5-8}$. With ESWL, a transducer generates high-amplitude pressure waves outside the body which are focused to the stone position where a shockwave is formed ${ }^{9,10}$. The stone comminution is caused by a combination of mechanical interactions such as pressure, tensile and shear forces, as well as spallation and cavitation ${ }^{2,11}$.

Compared to stone disintegration by ESWL, tumor ablation by HIFU is still a rather new treatment method but is likely to become increasingly important in the future ${ }^{3,12,13}$. Focused ultrasound waves are generated outside the body and pass the skin and the upper tissue layers without harming them ${ }^{4}$. With the help of real-time magnetic resonance imaging (MRI) the tumor tissue is brought into the focal region ${ }^{14}$, where the temperature is increased above $60^{\circ} \mathrm{C}$, thus leading to cell death by coagulative necrosis ${ }^{3}$. In addition to this immediate destructive effect due to heating of cells, ultrasound and shock waves can also transiently permeabilize living cells, which allows for applications in drug delivery and gene transfection ${ }^{4,15-17}$.

A different method for generating single pressure waves (in contrast to HIFU and ESWL) is based on the irradiation of an absorbing material with a pulsed laser ${ }^{18-21}$. With this technique, well-defined single pressure pulses are induced that propagate through the absorber material. Several studies have reported damage and permeabilization of mammalian cells, which were exposed to laser generated pressure waves ${ }^{22-28}$. It has also been demonstrated that laser induced pressure waves can be used for transdermal insulin delivery without causing any pain $^{26}$. One major advantage of using a laser for the generation of pressure waves is the very high reproducibility of the pressure conditions and the spatial precision of wave generation in contrast to transducer pressure waves ${ }^{29}$. In the former case, the pressure profile exhibits no negative pressure contributions, whereas in the latter case the negative part of the pressure profile leads to cavitation effects, which are very hard to control experimentally ${ }^{30-32}$. In addition, it has been shown, that pressure waves interacting with cells within time intervals of nanoseconds do 
not lead to any significant increase of temperature ${ }^{33,34}$. Thus, in contrast to HIFU, any destructive effect, observed as a consequence of the exposure of cells to a laser-generated pressure pulse with high gradient, is of purely mechanical nature. This makes laser induced pressure waves an ideal technique to study pressure effects on cells in cell culture.

In the study presented here, we systematically analyze a simple, versatile setup to investigate the effects of laser induced pressure waves on living cells. We use a combined experimental and theoretical approach to characterize the dynamic pressure conditions that the cells are exposed to. In many applications needle hydrophones are used to characterize dynamic pressure conditions in a fluid environment $^{25,28,35,36}$. However, the dimensions of the sensitive element of a hydrophone (diameter of approx. $500 \mu \mathrm{m}$ ) are considerably larger than the typical dimensions of biological cells (with diameters of approx. $15-150 \mu \mathrm{m})$.

To overcome this difficulty we use an optical high-speed velocimetry method to measure the velocity profile of the surface on which the cells are adherently grown ${ }^{37}$. These profiles serve as basic input for molecular dynamics simulations of the pressure wave propagation through the bottom of the multi-well plate, by which we can determine the pressure conditions on the relevant length and time scales, i.e. on the scale of micrometers and nanoseconds.

In order to demonstrate that our experimental setup is suitable for studying biological effects of pressure waves on cells, we present here an experimental study of the behavior of U87 glioblastoma cells, exposed to laser-induced mechanical pressure waves. U87 is a wellestablished cancer cell line which has been the subject of many biological studies ${ }^{38,39}$. The U87 cell line is not only a well-characterized cell culture model system ${ }^{40}$, but has also been frequently used to predict biological effects under in-vivo conditions ${ }^{41-43}$. In our study presented here, we demonstrate the destruction of U87 glioblastoma cells by laser-induced mechanical pressure waves and determine the corresponding pressure threshold for this biological effect to occur.

\section{Results}

To guarantee reproducible growth conditions for the cultivation of cells we use common microtiter plates as culture vessels where the pressure waves are generated. In these standard culture vessels the cells can be grown adherently prior to the experiments in a welldefined way. To optimize the conversion of laser energy into pressure waves, we use an absorber material at the outside of the bottom of the plate that is tightly connected for better transmission of the wave $e^{18-20}$. To achieve this, we coat the microtiter plate with black acrylic varnish that effectively absorbs the light of a Nd:YAG solid state laser (wavelength: $\lambda=1064 \mathrm{~nm}$ ). We additionally use a polymerization adhesive to bond a plate of transparent polymethylmethacrylate (PMMA) to the bottom facing the incoming laser beam. This confinement of the absorbing area further increases the peak pressure and thus generates even stronger pressure pulses. Upon illumination of the absorbing material and creation of the pressure wave, the absorber is burned. Thus, in every well, a pressure wave can only be generated once. For multiple measurements, we have to move to the next well (or use another plate).

To analyze the pressure waves generated by the laser we use a needle hydrophone, a measuring device that is commonly used for pressure wave characterization ${ }^{25,28,35,36,44}$. Our experimental setup is shown schematically in Fig. 1 . The hydrophone position $\mathrm{z}$ within the well can be varied with a motorized translation stage. We use the output of the Q-switch unit of the laser to trigger the measurement of the pressure profile with the hydrophone. Figure 2 a shows examples of these pressure profiles recorded at different hydrophone positions. It can be seen that the pressure rises to the peak pressure are very steep (within 10-20 ns) and that the pressure waves decay with time constants of $\tau=150 \mathrm{~ns}$. Each pressure wave is followed by a smaller wave with an approximate delay of $t_{d}=1350 \mathrm{~ns}$. The thickness of the

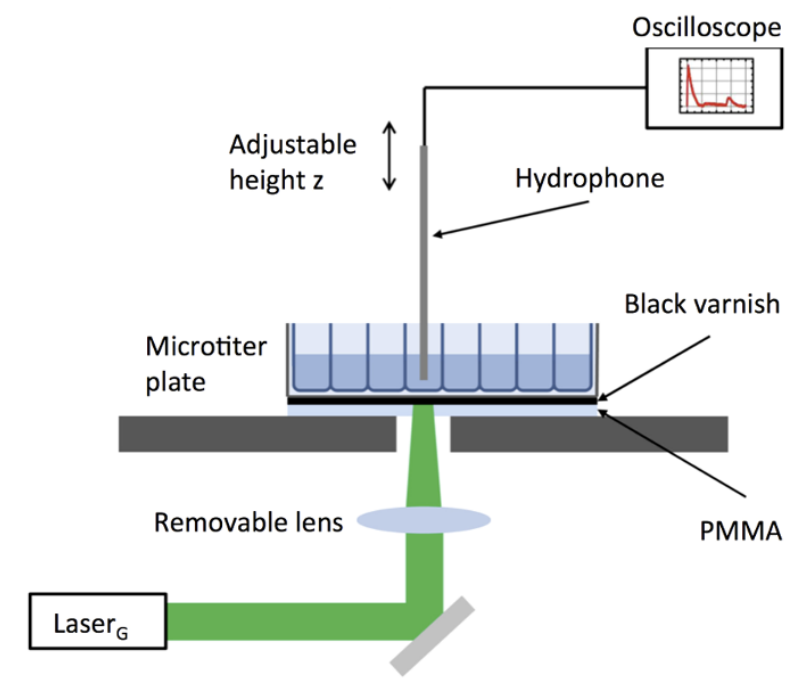

Figure $1 \mid$ Experimental setup for pressure wave characterization in water. The bottom of the microtiter plate is coated with an absorbing black varnish. In addition, the black surface can be covered with a transparent PMMA plate. To generate the laser-induced pressure waves, the microtiter plate is placed on a stage with a hole. A mirror guides the light of the laser (Laser $_{\mathrm{G}}$ ) onto the bottom of one well. A removable lens allows us to focus the beam. To guarantee that the entire well bottom is illuminated with the laser, we use 96 well plates when the focusing lens is in the beam path and 48 well plates when we do not focus the laser. The beam diameter of Laser $_{G}$ is approximately $9 \mathrm{~mm}$ and the diameter of a well in a 96 well plate is $6 \mathrm{~mm}$. We use a needle hydrophone to record pressure profiles inside the water filled well. The position of the hydrophone $\mathrm{z}$ can be adjusted with a motorized actuator.

well plate bottom is $x=1.5 \mathrm{~mm}$. Thus, this second pressure wave originates from reflections within the well bottom, assuming a speed of sound for polystyrene of $c_{P S}=2 x / t_{D}=2200 \mathrm{~m} / \mathrm{s}$. Interestingly, the pressure peak is not followed by a tensile (negative) pressure wave.

By varying the position of the hydrophone, we determined the velocity of the pressure wave by plotting the hydrophone position vs. the time of arrival of the pressure maximum at the hydrophone tip (Supplementary Fig. S1). We found that the velocity is constant at a value close to the speed of sound in water $\mathrm{C}_{\mathrm{H}_{2} \mathrm{O}} \approx 1500 \mathrm{~m} / \mathrm{s}$.

The setup contains a removable lens that focuses the laser beam on the absorbing surface (Fig. 1). To guarantee that the entire well area is exposed to the same pressure conditions, we use 96 well plates (with a well diameter of $d=6 \mathrm{~mm}$ ) when the laser beam is focused, and 48 well plates $(d=10 \mathrm{~mm})$ when the beam is unfocused. In order to test the effect of the focusing and using the PMMA cover, we recorded pressure pulses at the same positions $(z=2 \mathrm{~mm})$ inside the wells with varying laser pulse energies. The size of the irradiated area can easily be determined because the black varnish is burned upon laser illumination leading to a brown spot. Figure $2 \mathrm{~b}$ shows the peak pressure of the signals as function of the laser fluence $\phi=E / A$ (pulse energy per unit area) for the different configurations with or without cover and with or without focusing the beam. From Fig. $2 \mathrm{~b}$ we can quantify that the PMMA cover adhesively bond to the absorbing surface enhances the pressure waves by more than a factor of two in our setup. The data in Fig. 2b) show deviations for small laser fluences and peak pressures. However, this is not important for our results with cells, because we always perform cell experiments at peak pressures beyond $80 \mathrm{MPa}$. From Fig. $2 \mathrm{~b}$ one can see how to use the laser pulse energy to set the peak pressure of the pressure wave to a desired value. 
a

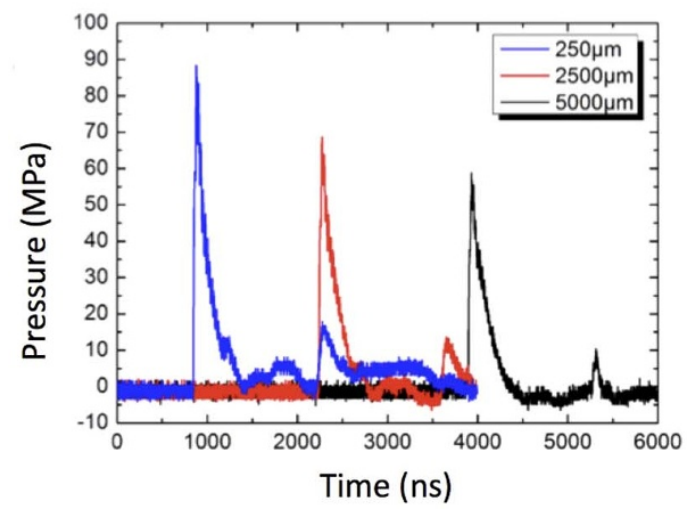

b

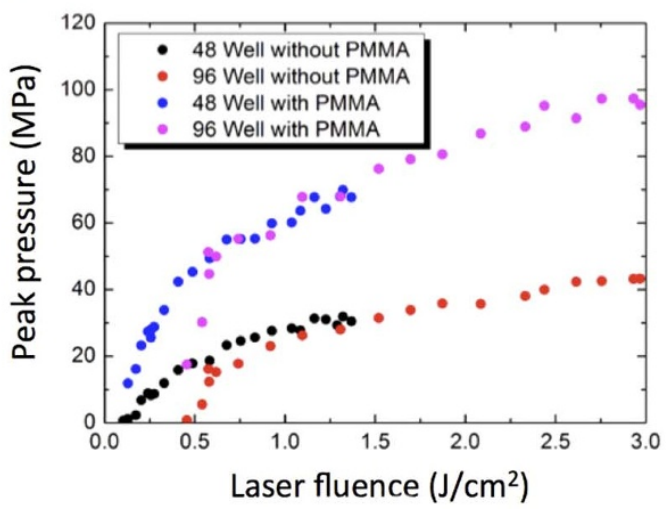

Figure $2 \mid$ Characterization of the pressure conditions with a hydrophone. We used the trigger output of the laser to start recording hydrophone data at time $t=0$. The position of the hydrophone in each well was adjusted with a motorized stage actuator (see Fig. 1). Each well was used for only one laser shot. (a) The pressure profiles shown were recorded at position $z=250 \mu \mathrm{m}$ (blue), $z=2500 \mu \mathrm{m}$ (red) and $z=5000 \mu \mathrm{m}$ (black), where $z$ is the distance between the bottom of the well and the hydrophone tip. The pressure waves were induced in a 48 well microtiter plate with PMMA cover without focusing the laser. (b) At a fixed hydrophone position $z=2000 \mu \mathrm{m}$ we extracted the maximum pressure $p_{\max }$ of the pressure wave for different laser pulse energies. In this plot we show the peak pressure as function of the laser fluence $\phi=E / A$ (pulse energy per unit area). The red and black dots were recorded in 96 and 48 well plates not covered with PMMA, respectively. The magenta and blue dots show peak pressures in 96 and 48 well plates with PMMA cover, respectively.

Characterizing the pressure waves with a hydrophone has two major disadvantages. (i) The sensitive area of the hydrophone (diameter of approx. $500 \mu \mathrm{m}$ ) that measures the pressure is large compared to a cell. As mentioned above, we make sure that the full area of the well bottom is exposed to the same pressure conditions. However, the pressure condition could change in the direction of wave propagation. In particular, it was shown that pressure waves in similar setups decay within few micrometers ${ }^{22,45-47}$. Thus, pressure information at a better spatial resolution would be desirable. (ii) In order not to break the sensitive sensor in the tip, the hydrophone cannot touch a surface. However, as we observe the impact of pressure waves on an adherently grown monolayer of cells (see below) we are particularly interested in the pressure conditions in direct vicinity of the well bottom.

For the characterization of the pressure wave close to the well bottom, we take advantage of the fact that the reflection of a pressure wave at a free surface is accompanied by an acceleration of the surface. The acceleration results from fundamental physical conservation laws and from the boundary condition of zero pressure at the surface. An approximate analytic expression for the relation between surface velocity $v_{s}$ and pressure $p$ is given in Zel'dovic et al. ${ }^{48}$ :

$$
p=\frac{1}{2} v_{s} \rho_{0} c_{0}
$$

where $\rho_{0}$ is the density of the fluid and $c_{0}$ is the speed of sound. Thus, measurement of the surface velocity profile yields information of the pressure of the reflected wave.

For the characterization of the velocity profile, the technique of photon Doppler velocimetry (PDV) was used. A schematic drawing of the setup of the PDV is shown in Fig. 3 and a detailed description of the PDV measurements as well as the calculation of the pressure from the measured velocity profiles are presented in the Methods section.

Figure $4 \mathrm{a}$ shows the velocity profile of the well bottom surface at the maximum laser pulse energy of $E=0.87 \mathrm{~J}$. The velocity exhibits a sharp increase up to $v_{s}=150 \mathrm{~m} / \mathrm{s}$ followed by a smooth decay. Similar to the pressure profiles recorded with the hydrophone (Fig. 2a), the first velocity peak is followed by a second peak after $1300 \mathrm{~ns}$. As discussed above, this second acceleration of the surface is due to reflections of the pressure wave in the well bottom. To further quantify the influence of the Laser pulse energy on the resulting pressure wave, we recorded surface velocity profiles at different energies ranging from $E=0.87 \mathrm{~J}$ to $E=0.37 \mathrm{~J}$. The maximum velocity changes with laser energy while the decay time constant seems to be independent of pulse energy (Fig. 4b).

The velocity profiles from Fig. $4 \mathrm{~b}$ were used as input for numerical simulations of the movement of the pressure wave through the water in the well. A discussion of the numerical method is given in the Methods section. Briefly, the velocity of the well bottom rises sharply to a value $v_{0}$ and then decreases with an exponential decay function with time constant $\tau$. We extracted $v_{0}$ and $\tau$ from fits to the PDV data (Supplementary Fig. S2). Next, we calculated the peak pressure at different positions in the well. Here, we made sure that the choice of the spatial discretization does not affect the results (Supplementary Fig. S3). In Fig. 5a-e we show the numerically calculated peak pressure as a function of position for different laser pulse energies (red lines). These numerical results are in good agreement with the measured hydrophone data (black dots). Thus, the simulation results seem correct for large distances to the cells. This indicates that we also may extract realistic pressure conditions from simulations in direct vicinity of the well bottoms (where a measurement is impossible).

Having characterized the physical properties of the pressure waves that we generate in our proposed setup, we tested their effect on living cells. In particular, we determined the threshold of pressure that results in irreversible damage of the cells. As shown above, the pressure conditions are well reproducible and the Q-switch delay unit of the laser can be used to set the peak pressure.

To test which peak pressure is needed to kill U87 brain cancer cells we used two different techniques to determine the cell concentration: The Coulter principle and the trypan blue staining method ${ }^{49}$. Since we are interested in the destructive effect of the pressure waves, we carried out experiments in 96 well plates with PMMA cover. In this setup, we get the highest peak pressures (Fig. 2) that can be tuned with the laser pulse energy. Each experiment was carried out in the following way: The cells were grown in the prepared microtiter plates for 2-3 days. We then exposed several wells to single laser pulses. Subsequently, we determined the number of living cells found in the cell layer (i.e. not in the supernatant) of a treated well $n_{\text {live }}^{t r}$ and the number of living cells found in the cell layer of the untreated well $n_{\text {live }}^{\text {untr }}$. Figure 6a shows the ratio $n_{\text {live }}^{\text {tr }} / n_{\text {live }}^{\text {untr }}$ for different laser pulse 


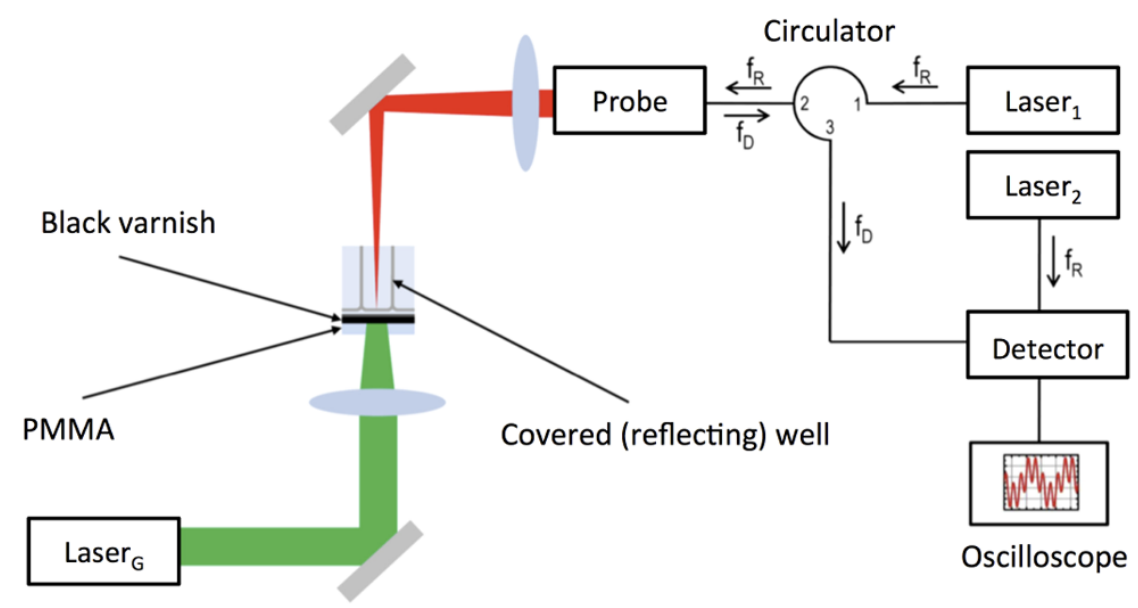

Figure 3 Experimental setup for determination of the velocity profile of the well bottom. The pressure waves are generated upon illumination of the microtiter plate by Laser $_{\mathrm{G}}$ (see Fig. 1). For the velocity measurements we used a high-speed velocimetry system based on the heterodyne method described in ref. 37. With this technique the velocity of a moving surface is calculated from the Doppler-shifted frequency $f_{D}$ of light that is reflected by the surface. We use two lasers with a frequency $f_{D}=193.414 \mathrm{GHz}$. The illumination laser ( Laser $_{1}$ ) is guided to a probe with an optical fiber and focused unto the microtiter plate that was covered with a reflecting chrome/silver coating. The reflected light (with the Doppler shifted frequency $f_{D}$ is collected by the same probe and transported to the detector. At the detector, the Doppler shifted light and the light of the reference laser ( Laser $\left._{2}\right)$ are mixed to record the beat frequency $f_{B}$ of the resulting signal $I(t)$. To determine the velocity profile of the well bottom that results from arrival of the pressure wave, we started to record $I(t)$ at the moment of pulse generation with $\operatorname{Laser}_{\mathrm{G}}$.

energies. Thus, Fig. 6a shows that with the maximum pulse energy of $E=0.87 \mathrm{~J}$, less than $20 \%$ of the cells survive this treatment.

To further clarify the impact of the pressure wave on the treated sample, we also analyzed the supernatant in the wells and determined the number of living and dead cells with the hemocytometer. In the treated sample, $n_{\text {dead }}^{t r}$ denotes the number of dead (trypan positive) cells in the cell layer, and $m_{\text {dead }}^{\text {tr }}$ and $m_{\text {live }}^{\text {tr }}$ denote number of dead and living cells in the supernatant, respectively. The total number of cells in the treated sample is thus given by $n_{\text {tot }}^{t r}=\left(n_{\text {live }}^{t r}+n_{\text {dead }}^{t r}+m_{\text {live }}^{t r}+m_{\text {dead }}^{t r}\right)$. Correspondingly, the total number of cells in the untreated sample is $n_{\text {tot }}^{\text {untr }}=\left(n_{\text {live }}^{\text {untr }}+n_{\text {dead }}^{\text {untr }}+m_{\text {live }}^{\text {untr }}+m_{\text {dead }}^{\text {untr }}\right)$, where $n_{\text {dead }}^{\text {untr }}$ is the number of dead cells in the cell layer, and $n_{\text {dead }}^{\text {untr }}$ and $n_{\text {live }}^{\text {untr }}$ denote the number of dead and living cells in the supernatant.

It turned out that for all samples most of the dead cells are found in the supernatant rather than in the cell layer, i.e. $m_{\text {dead }}^{\text {tr }} \gg n_{\text {dead }}^{\text {tr }}$. The red dots in Fig. $6 \mathrm{~b}$ show $m_{\text {dead }}^{\text {tr }} / n_{\text {tot }}^{\text {untr }}$ for varying Laser pulse energy. It is obvious that for the highest pulse energy $E=0.87 \mathrm{~J}$ the number of dead cells in the supernatant $\left(m_{\text {dead }}^{\text {tr }}\right)$ cannot fully explain the low number of cells found in the cell layer $n_{\text {live }}^{\text {tr }}$ (see Fig. 6a). We therefore also calculated the total number of cells found in the treated sample divided by the total number of cells found in the untreated sample $n_{\text {tot }}^{\text {tr }} / n_{\text {tot }}^{\text {untr }}$ (black dots in Fig. 6b). Interestingly, for the highest pule energy we find $n_{\text {tot }}^{\text {tr }} / n_{\text {tot }}^{\text {untr }} \approx 0.5$, thus $50 \%$ of the cells seem to be completely destroyed (and are not found as trypan positive cells in the hemocytometer). As a measure for the pressure threshold upon which cell destruction occurs we thus define the pressure level at which at least $50 \%$ of the cells have been destroyed by the treatment.

In summary, from the data in Fig. 6 we see that for the strongest pressure pulses (i) only a few ( $\leq 20 \%$ ) cells of the cell layer survive, (ii) most of the dead cells are found in the supernatant, and (iii) a large fraction of cells seem to be completely destroyed. To double check the last point, we analyzed the volume distributions measured by a a

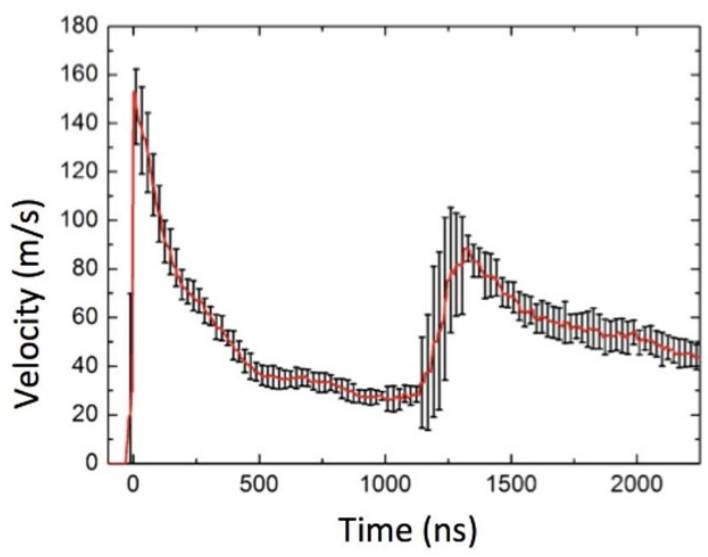

b

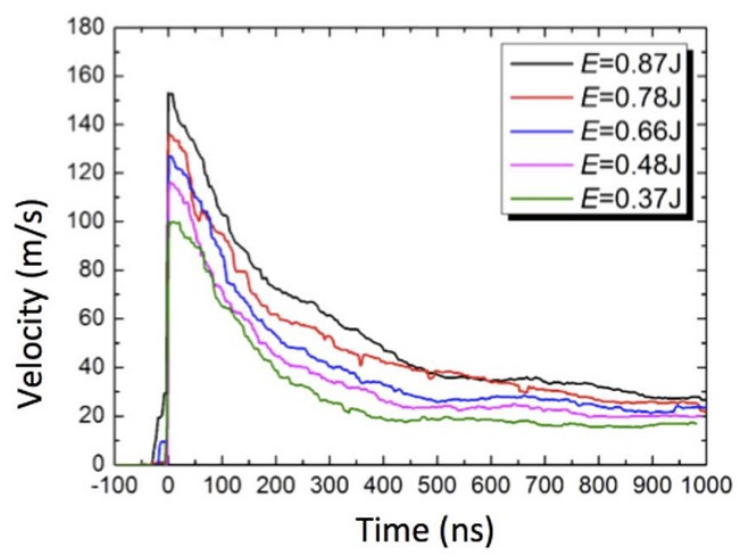

Figure 4 | Velocity profiles measured with the PDV method. We used the setup shown in Fig. 3 to determine the velocity profiles of the well bottom due to the pressure wave propagating through it. Shown are results in 96 well plates with PMMA cover. We recorded 15 single velocity profiles, shifted the maximum to the position $t=0$ and averaged. (a) Averaged profile for the maximum pulse energy $E=0.87 \mathrm{~J}$; error bars are standard deviations. (b) Averaged velocity profiles for different laser pulse energies ranging from $E=0.83 \mathrm{~J}$ to $E=0.87 \mathrm{~J}$. 
a

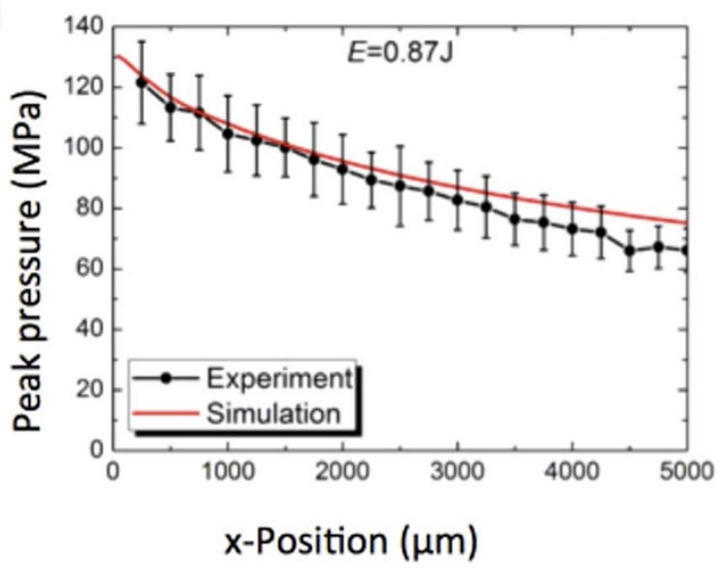

C

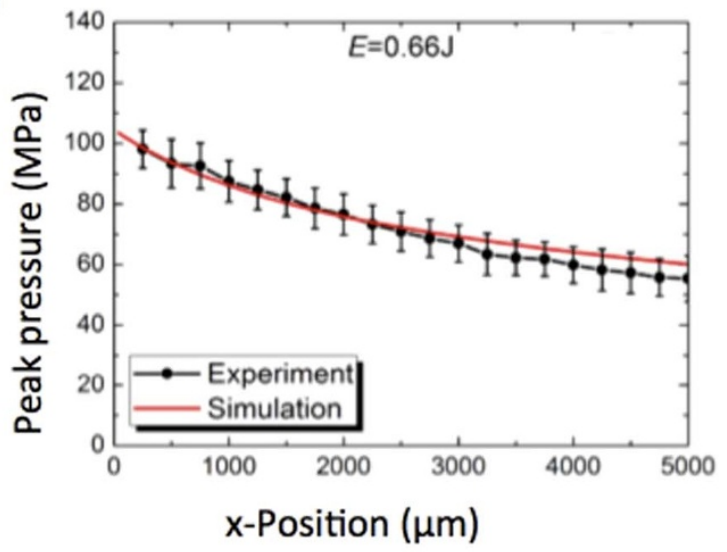

b

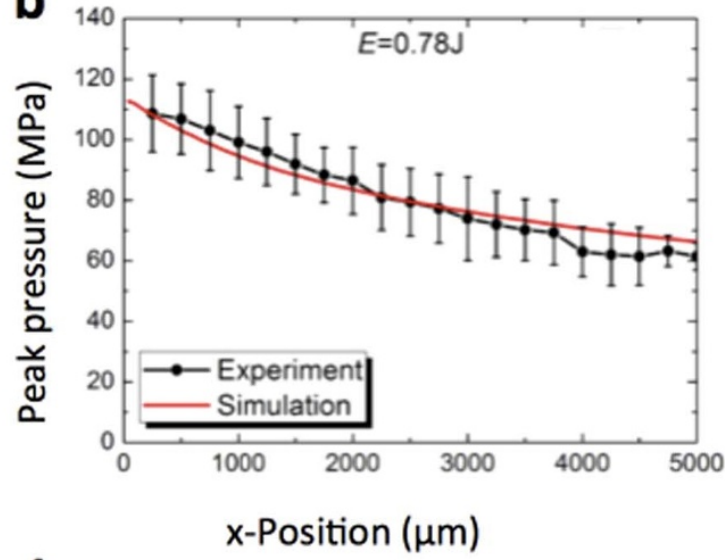

d

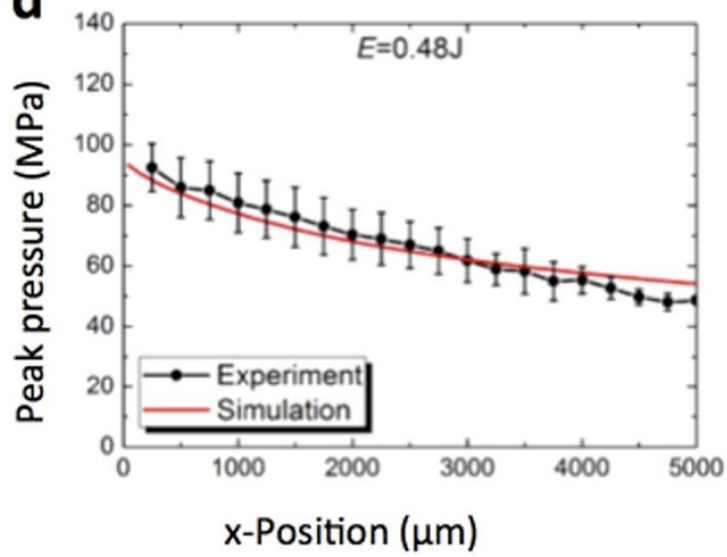

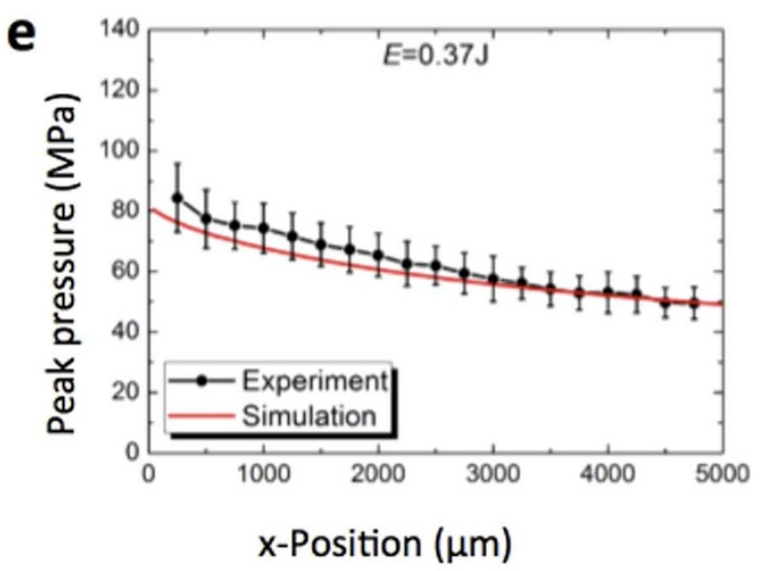

Figure 5 Peak pressures inside the well calculated from numerical simulations and comparison to hydrophone signals. We used the velocity profiles of the well bottom (see Fig. 4a-e) as input to simulate the pressure conditions inside a well at different laser pulse energies. At constant time intervals the peak pressure and the position of the pressure wave were calculated in numerical simulations (red lines). The black dots show the peak pressure at different positions inside the well as extracted from hydrophone data (see Fig. 2). Each data point represents the average of five independent measurements at different plates; error bars display standard deviations.

Coulter Counter of a sample treated at the maximum laser pulse energy (Fig. 7). For comparison, the green and the black lines show the volume distributions of the cell layer and the supernatant of an untreated reference sample, respectively. As expected there are hardly any particles found in the untreated supernatant (black curve). The volume distribution of the untreated cell layer (green curve) also has the expected form with a mean volume of $1.75 \mathrm{pl}$. This volume corresponds to an approximate cell diameter of $15 \mu \mathrm{m}$, which is in agreement with the microscopy images (Supplementary Fig. S4).

In the treated sample, the volume distribution of the cell layer (blue line in Fig. 7) has the same form as the untreated cell layer but with a smaller total number of cells. The supernatant of the treated sample (red line in Fig. 7) on the other hand shows a large peak of small particles (debris). Thus, these volume distributions confirm that the cells were destroyed and detached from the surface by the pressure wave.

\section{Discussion}

In this work we have introduced a simple method to expose living cells to pressure waves under well-reproducible physical conditions. Since the cells grow in standard culture vessels (here we used microtiter plates) also the growth conditions are well defined. We have 
a

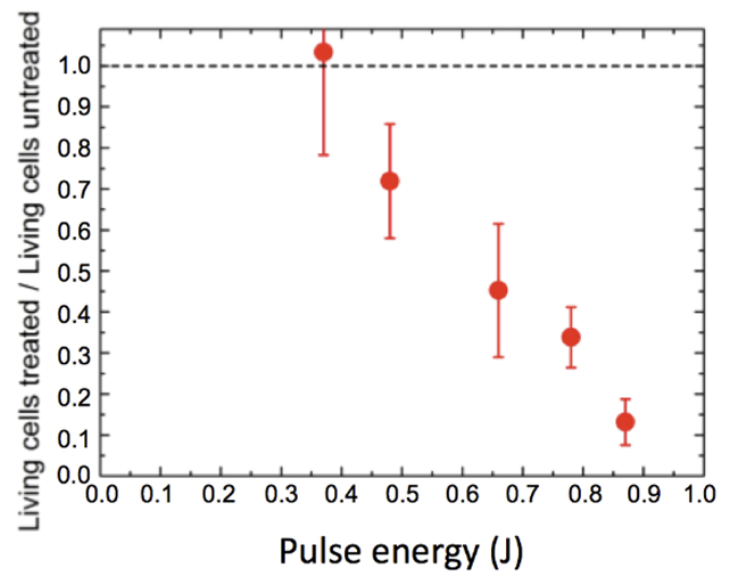

b

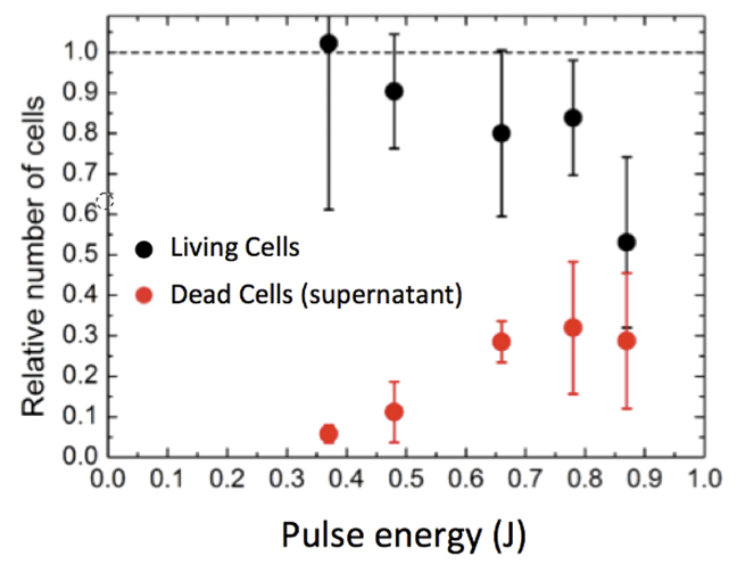

Figure 6 Analysis of cell survival after exposure to pressure wave. Prior to pressure wave experiments, the U87 cells were grown in the prepared microtiter plate for several days (for details see Methods Section). In 15 wells the cells were treated with pressure waves while the cells in 19 wells of the same plate served as the untreated reference. Subsequently, the concentration of cells was determined with a hemocytometer by trypan blue exclusion. (a) Number of cells found in the cell layer of the treated sample divided by the number of cells found in the untreated reference, $n_{\text {live }}^{\text {tr }} / n_{\text {live }}^{\text {untr }}$. With the hemocytometer we also determined the numbers of dead cells in the cell layer, $n_{\text {dead }}^{\text {tr }}$ and $n_{\text {dead }}^{\text {untr }}$, as well as the number of living $\left(m_{\text {live }}^{\text {tr }}, m_{\text {live }}^{\text {untr }}\right)$ and dead $\left(m_{\text {dead }}^{\text {tr }}, m_{\text {dead }}^{\text {untr }}\right)$ cells in the supernatant. (b) Total number of cells found in the treated sample divided by the total number of cells found in the untreated sample, $n_{\text {tot }}^{\text {tr }} / n_{\text {tot }}^{\text {untr }}=\left(n_{\text {live }}^{\text {tr }}+n_{\text {dead }}^{\text {tr }}+m_{\text {live }}^{\text {tr }}+n_{\text {dead }}^{\text {tr }}\right) /\left(n_{\text {live }}^{\text {untr }}+\right.$ untr $\left.+m_{\text {live }}^{\text {untr }}+n_{\text {dead }}^{\text {untr }}\right)$ (black dots) and the relative number of dead cells found in the supernatant, $m_{\text {dead }}^{\text {tr }} /\left(n_{\text {live }}^{\text {untr }}+n_{\text {dead }}^{\text {untr }}+m_{\text {live }}^{\text {untr }}+n_{\text {dead }}^{\text {untr }}\right)$ (red dots). All data shown were recorded in 96 Well plates with PMMA cover. Data points are averages of four independent measurements, error bars are standard deviation.

systematically characterized the physical properties of the pressure waves with experimental and theoretical methods. As a first result with respect to a biological effect, we determined the peak pressure needed to destroy U87 brain cancer cells. Based on a 50\% survival rate in Figure $6 \mathrm{a}$ ) the threshold pulse energy of our laser to destroy the cells lies at $0.65 \mathrm{~J}$, which, along with the data presented in Figure $2 \mathrm{~b}$ ) corresponds to a peak pressure of $80 \mathrm{MPa}$.

For the analysis of cell damage, we used two different standard methods to determine cell concentrations, trypan blue exclusion and the Coulter principle ${ }^{49}$. While the trypan blue exclusion method allows for determining the concentration of dead and living cells

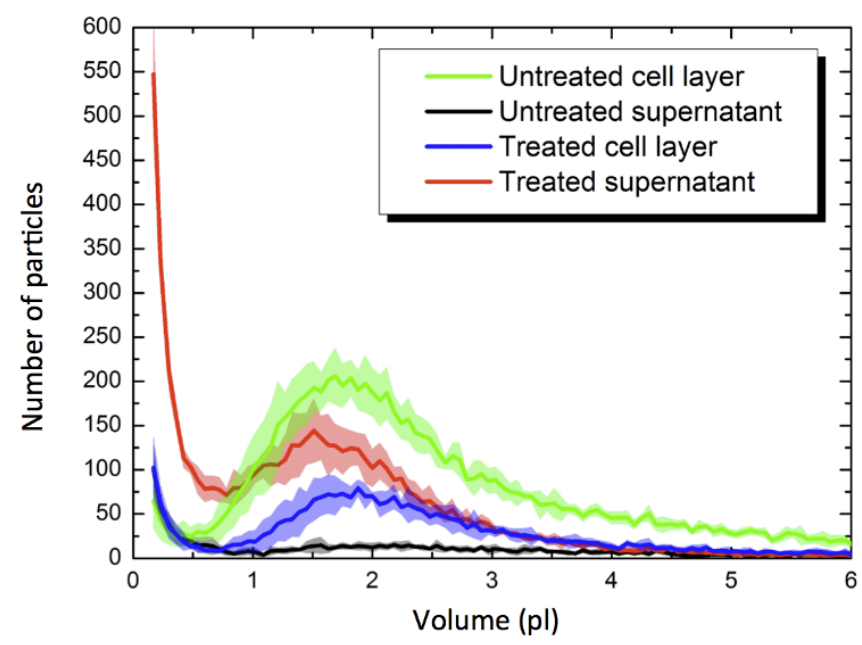

Figure 7 Volume distributions of cell layer and supernatant. We use a Counter to determine the volume distributions of particles in the treated sample (blue and red line) and in the untreated reference (green and black line). Each curve is an average of five measurements at the same sample. The error bars (shown as lines with fill areas) are standard deviation. The pressure wave was generated at the full laser pulse energy of $E=0.87 \mathrm{~J}$ in a 96 well plate with PMMA cover. separately, the Coulter counter measures the volume distribution of particles, where cell debris can be seen. In other words, combining both techniques one can distinguish living, dead and completely destroyed cells. In this way we were able to show that the strongest pressure waves detached the cells form the substrate and destroyed up to $50 \%$ of them completely (Fig. 6 and Fig. 7). A similar behavior is seen when cells are exposed to collapsing cavitation bubbles ${ }^{3,50-52}$. In our experiments however, this effect is not due to cavitation because there is no negative (tensile) pressure in the pressure profiles (Fig. 2). Several studies reported damage of different cell types treated by laser-induced pressure waves generated in similar setups ${ }^{23-25}$. In our study we quantify that less than $20 \%$ of U87 brain cancer cells survive exposure to pressure waves with $p_{\max }=130 \mathrm{MPa}$ (Fig. 5 and Fig. 6). The peak pressure can be tuned by the pulse energy per unit area of the laser (Fig. 2). The maximum peak pressure generated in our setup without focusing the beam (using 48 Well plates) is $p_{\max }=$ $90 \mathrm{MPa}$. We have shown that the cells survive this exposure by determining that $n_{\text {live }}^{\text {tr }} / n_{\text {live }}^{\text {untr }}=0.94 \pm 0.12$. As seen in Fig. 6a, also when the beam is focused (but the pulse energy is reduced accordingly) all cells survive. We note the values for $p_{\max }$ are determined from the simulation in direct vicinity of the growth surface and not by a hydrophone.

The use of a hydrophone for determining the pressure conditions in a fluid has the major drawback that it cannot touch the surface where the cells actually grow. The minimum distance to the well bottom is $z=250 \mu \mathrm{m}$ in order to protect the sensitive hydrophone tip. To determine the conditions that the cells are exposed to on the microscopic level, we use the optical PDV method ${ }^{37}$. Our experimental results demonstrate that PDV is well suited for the investigation of highly transient processes associated with the reflection of the pressure wave and that it allows for the measurement of the velocity profiles of the well bottom with a temporal resolution on the order of nanoseconds. By covering the transparent polystyrene of the microtiter plates with a chrome/silver coating the surface is diffusely reflecting and thus allows for detection of the PDV signal.

The measured velocity profiles of the surface can be used as input for a numerical molecular dynamics simulation of the pressure wave 
propagation through the water, also in very close vicinity of the well bottom. The calculated peak pressures of the propagating pressure wave in the computer simulation at different positions in the well are in agreement with corresponding hydrophone signals (Fig. 5). As mentioned above, on the timescale of nanoseconds, cells do not increase their temperature while a pressure wave passes, thus the observed killing of cells is a pure mechanical effect and not due to heating of cells as in HIFU applications.

The pressure wave propagates at a constant velocity that is close to the speed of sound in water. Thus, strictly speaking, it is not a shock wave (which would move faster than the speed of sound in the respective medium). As reported in the literature the laser creates a shock wave when it is absorbed ${ }^{18-21}$. However a shock wave decays very fast (after a few $\mu \mathrm{m})$ in water and tissue $\mathrm{e}^{22,45-47}$. As shown in this study (Supplementary Fig. S1 and Supplementary Fig. S2), the shock wave has already decayed to an ordinary pressure wave when it enters the interior of the well and interacts with the cells. Thus, the effects that are seen here are no shock wave effects. As seen in Fig. 5 the peak pressure decreases while the pressure wave traverses the fluid. However, at a comparably large distance of $\mathrm{z}=5000 \mu \mathrm{m}$ the peak pressure is still over $p_{\max }=70 \mathrm{MPa}$. This comparably weak decay is a prerequisite for a possible long-term application in organisms. For example, similar to HIFU ${ }^{3,4}$ and ESWL ${ }^{1,2}$ applications, it should be possible to create the pressure wave outside the body with subsequent focusing of the wave and thus have the strongest effect in the focus region inside the body. In the future, in-vivo experiments together with simulations based on real tissue properties could show if such an application is possible. Knowing that the pressure conditions at a particular position in the body are the same as in this study, our cell culture experiments could predict the in vivo effect. In a study in Ref $^{23}$ it was shown that injury in mouse breast sarcoma cells after being exposed to 5 repeated pressure pulses was better related with the rise time of the generated pressure waves than with the peak pressure. However, the results of this study were obtained under very different conditions than in our experiments. We only use very reproducible single pressure pulses to investigate cell damage as our studies have shown that using repetitive shots at a target material changes the resulting pressure waves and thus renders the experiment less controllable. Nevertheless, a systematic study of the influence of rise times on the damage or killing of cells would be of great interest, but requires a laser system with variable pulse duration. In our case, the pulse duration is fixed at $\sim 6 \mathrm{~ns}$.

In conclusion we have shown that it is possible to generate strong pressure waves in a versatile setup with standardized growth conditions for cells. We have very thoroughly and systematically characterized the physical properties of the pressure waves. We carried out state-of-the-art PDV experiments to determine the velocity profile of a surface and used the obtained velocity profile as input for numerical pressure simulations. These simulations can determine the pressure conditions in the direction of shock wave propagation on cellular length scales. For lithotripter induced shock waves, the damage threshold concerning peak pressure and the number of shocks needed to induce damage varies significantly for different cell types ${ }^{9,47,53,54}$. Thus, in future studies it will be interesting to test the behavior of different cell types in this setup and systematically compare cell damage effects. In addition, the exact mechanism of cell damage on the subcellular or even molecular level should be studied. Here, microscopic and spectroscopic methods will be able to provide interesting insights. As in our proposed setup, due to the small timescale involved, the interaction of the pressure waves with cells is of purely mechanical nature (in contrast to heating with HIFU applications), one should also test mechanical properties of the cells for example with atomic force microscopy (AFM).

As mentioned above, in our setup the cells survive pressure waves with $p_{\text {max }}=90 \mathrm{MPa}$, a comparably large pressure. Here the question arises what properties of these cells might be altered after pressure wave exposure. For example, as discussed above, it is possible to permeabilize living cells with pressure waves giving rise to transfection applications ${ }^{16}$. A permeabilization may also have possible applications in drug delivery ${ }^{15}$. For these applications it is essential that the pressure wave decay is not too strong. Hence, our experimentally well-reproducible system introduced here may provide a good basis for the test of biological effects of pressure waves.

\section{Methods}

Pressure wave generation. The bottom of each microtiter plate was coated with black acrylic gloss varnish (C.Kreul GmbH, Hallerndorf, Germany) and left to cure for at least 48 hours. Subsequently, $5 \mathrm{~mm}$ thick transparent PMMA plates are bonded to the varnish surface with the ACRIFIX 192 polymerization adhesive (Evonik Degussa, Essen, Germany). The pressure waves were generated by illumination with a single 6 ns laser pulse with a wavelength of $1064 \mathrm{~nm}$ (Brilliant B Q-switched Nd:YAG Laser, Quantel, Erlangen, Germany). The laser pulse energy was varied with the Q-Switch delay unit. We used a power meter (Thorlabs S370C) to determine the laser power at constant laser pulse generation at $10 \mathrm{~Hz}$ to calculate the pulse energy at the sample position for varying delay time. To record the stress waves in water we used MüllerPlatte needle hydrophones (Dr. Müller Instruments, Oberursel, Germany) mounted on a motorized translation stage with a bidirectional repeatability of $1.6 \mu \mathrm{m}$ (see Fig. 1). The hydrophone signals were recorded with a Tektronix TDS 5054 digital phosphor oscilloscope and transformed to pressure profiles using the sensitivity given by the producer.

Determination of the velocity profiles. The reflection of the pressure wave at the well bottom is accompanied by an acceleration of the surface. As discussed above, the pressure of the reflected wave can be derived from the surface velocity profile. For the experimental measurement of the velocity profile of the well bottom, we used the technique of photonic Doppler velocimetry (PDV) described by Strand et al. ${ }^{37}$.

PDV is an optical technique that is based on the Doppler shift of the light reflected from a moving object and allows for a non-invasive measurement of velocities with high time resolution. For an enhancement of the PDV signal, the well bottom was covered with reflecting chrome/silver coating or with aluminum foil. Both procedures yielded the same results.

A drawing of the PDV setup for the measurement of the surface velocity profiles is shown in Fig. 3. The light of a cw (continuous wave) Erbium fiberlaser (referred to as illumination laser) with a maximum output power of $2 \mathrm{~W}$ is directed onto the surface of the sample by an optical probe. The wavelength $\lambda_{R}$ of the laser can be adjusted around the center wavelength of $\lambda_{R}=1557 \mathrm{~nm}$, corresponding to a frequency of $f_{R} c$ l $\lambda_{R}=200 \mathrm{THz}$ (where $c$ is the speed of light). The optical probe basically consists of a lens that focuses the light of the laser onto the surface of the sample. A fraction of the light that is diffusely or specularly reflected by the surface is collected by the same optical probe used for illumination and is separated from the illumination light by a circulator. The reflected light with a Doppler shifted frequency $f_{D}$ is guided to a high bandwidth optical detector where it is mixed with the light of a second $\mathrm{cw}$ laser (referred to as reference laser) with a maximum output power of $40 \mathrm{~mW}$ and with a frequency that is equal to the frequency of the illumination laser $f_{R}$. Both lasers exhibit a small bandwidth of less than $1 \mathrm{kHz}$ which is essential for a precise velocity measurement.

The frequency of the Doppler shifted light that is reflected from the moving surface is given by

$$
f_{D}=\frac{c+v_{s}}{c-v_{s}} f_{R}
$$

At the detector, heterodyne mixing of the electromagnetic fields of the Doppler shifted light and the light of the reference laser results in the formation of a beat signal with a frequency $f_{B}$ that is equal to the frequency difference of the superimposed light fields:

$$
f_{B}(t)=2 f_{R} \frac{v_{s}(t)}{c}
$$

The beat signal is recorded with a high bandwidth digital oscilloscope.

For the calculation of the velocity profile, the temporal evolution of the beat frequency has to be determined. To this end, a short time Fourier transform (STFT) algorithm similar to that described by Strand et al. ${ }^{37}$ was applied to extract the beat frequency from the measured signal. The application of the STFT implies that the achievable velocity resolution depends on the width of the STFT window function and thus on the time resolution. For a Gaussian window function, the relation between the standard deviation of the window function $\sigma_{t}$ and the standard deviation of the velocity $\sigma_{v}$ is:

$$
\sigma_{t} \sigma_{v} \geq \frac{\lambda_{R}}{8 \pi}
$$

For the presented measurements a STFT window size of $\sigma_{t}=32.8$ ns was chosen resulting in a velocity resolution of $\sigma_{v}=1.9 \mathrm{~m} / \mathrm{s}$.

Numerical simulations. Numerical simulations of propagating pressure waves in water were performed using the standard velocity-Verlet algorithm ${ }^{55,56}$ implemented in the open source molecular dynamics (MD) code LAMMPS ${ }^{57}$, which was used to 
carry out the simulations. The pressure wave was modeled by assigning the velocity profiles (see Fig. $4 \mathrm{~b}$ and detailed discussion above) as initial conditions for water particles that constitute the propagating pressure front. The water particles were modeled using a coarse-grained (CG) approach by mapping $4 \mathrm{H}_{2} \mathrm{O}$ molecules onto a single (CG) water particle according to the method described by Steinhauser et al ${ }^{58,59}$. All CG water particles were coupled to a DPDE ${ }^{60,61}$ (Dissipative Particle Dynamics at constant Energy) local thermostat, which couples the particles' velocities to an internal energy reservoir $e_{i}$. DPDE is a coarse-graining simulating technique for MD, which is implemented in LAMMPS and has recently been applied to biological systems $s^{58,59,62,63}$. It mimics the complex particle dynamics of a fully detailed system (e.g. a fluid) by assigning an internal energy variable $e_{i}$ to each particle $i$ and a heat capacity $C_{V}$, such that the temperature of the $i$-th particle is given by $T_{i}=e_{i} / C_{V}$. With this CG approach it is possible to measure the resulting pressure in the fluid at different positions on a lenght scale comparable to the size of a typical eukariotic cell which is not possible in experimental measurements using a needle hydrophone, due to the limitations discussed in detail above. The CG particles that constitute the pressure front are assigned the initial velocity profile $v_{s}$ and then start to transfer their momentum, i.e. their kinetic energy to neighboring particles, thus propagating the pressure front through the fluid. A velocity-Verlet time integration scheme with a timestep $\Delta t=4.89 \mathrm{fs}$ and a reference density of $1000 \mathrm{~kg} / \mathrm{m}^{3}$ and $c_{0}=1498 \mathrm{~m} / \mathrm{s}$ were used. Equation (1) finally allows for calculating the resulting pressure by averaging over the particle velocities at corresponding positions. The results are displayed in Fig. 5 (red lines) and show very good agreement with experimental hydrophone measurements.

Cell culture and analysis. U87 was graciously provided by A. Weyerbrock, Department General Neurosurgery, University Medical Center Freiburg, Germany. U87 were grown in DMEM (Gibco) supplemented with $10 \%$ fetal calf serum (FCS) and $1 \%$ antibiotics and antimycotics (Gibco).

For the pressure wave experiments, the microtiter plates were prepared as explained above. The cells were cultivated in these plates for 2 to 3 days before the experiments. Tests with non-prepared (transparent) microtiter plates showed that the cells were approx. $80 \%$ confluent after this period. When using 96 Well plates, 15 wells were exposed to one single laser pulse each. 19 Wells on the same plate served as the untreated reference. In experiments with 48 well plates, 6 wells were treated and 16 served as untreated reference. The cell layers were trypsinated with TrypLE (Gibco) and pooled before cell counting without centrifugation. For the cell counting with the hemocytometer, we used a 1:1 mixture of sample liquid with Trypan Blue. The supernatants were analyzed in the same way.

1. Lingeman, J. E., McAteer, J. A., Gnessin, E. \& Evan, A. P. Shock wave lithotripsy: advances in technology and technique. Nat. Rev. Urol. 6, 660-670 (2009).

2. Rassweiler, J. J. et al. Shock wave technology and application: an update. Eu. Urol. 59, 784-796 (2011).

3. Zhou, Y. F. High intensity focused ultrasound in clinical tumor ablation. World J. Clin. Oncol. 2, 8-27 (2011).

4. Coussios, C. C. \& Roy, R. A. Applications of acoustics and cavitation to noninvasive therapy and drug delivery. Annu. Rev. Fluid Mech. 40, 395-420 (2008).

5. McClure, S. \& Dorfmüller, C. Extracorporeal shock wave therapy: theory and experiment. Clin. Tech. Equine Pract. 2, 348-357 (2003).

6. Shrivastava, S. K. \& Ailash, K. Shock wave treatment in medicine. J. Biosci. 30, 269-275 (2005).

7. Benes, J. et al. Biological effects of two successive shock waves focused on liver tissues and melanoma cells. Physiol. Res. 56, S1-S4 (2007).

8. Rassweiler, J. J. et al. Shock wave technology and application: An update. Eu. Urol. 59, 784-796 (2011).

9. Coleman, A. J. \& Saunders, J. E. A review of the physical properties and biological effects of the high amplitude acoustic field used in extracorporeal lithotripsy. Ultrasonics 31, 75-89 (1993).

10. Delius, M. Medical applications and bioeffects of extracorporeal shock waves. Shock Waves 4, 55-72 (1994).

11. Thiel, M. Application of shock waves on medicine. Clin. Orthop. Related Res. 387, 18-21 (2001).

12. Kennedy, J. E. et al. High intensity focused ultrasound: surgery of the future? Brit. I. Raiol. 76, 590-599 (2003).

13. Ter Haar, G. \& Coussious, C. High intensity focused ultrasound: physical principles and devices. Int. J. Hyperther. 23, 89-104 (2007).

14. Hynynen, K. MRI-guided focused ultrasound treatments. Ultrasonics 50, 221-229 (2010).

15. Rodriguez-Devora, J. I. et al. Physically facilitating drug-delivery systems. Ther Deliv. 3, 125-139 (2012).

16. Yao, C. P., Zhang, Z. X., Rahmanzadeh, R. \& Huettmann, G. Laser-based gene transfection and gene therapy. IEEE T. Nanobiosci. 7, 111-119 (2008).

17. Sanches, P. G., Grull, H. \& Steinbach, O. C. See, reach, treat: ultrasound-triggered image-guided drug delivery. Ther. Deliv. 2, 919-934 (2011).

18. Bushnell, J. C. \& McCloskey, D. J. Thermoelastic stress production in solids. J. Appl. Phys. 39, 5541-5546 (1968).

19. Fabbro, R., Fournier, J., Ballard, P., Devaux, D. \& Virmont, J. Physical study of laser-produced plasma in confined geometry. J. Appl. Phys. 68, 775-784 (1990).
20. Yang, L. C. Stress waves generated in thin metallic-films by a q-switched rubylaser. J. Appl. Phys. 45, 2601-2608 (1974).

21. Zweig, A. D., Venugopalan, V. \& Deutsch, T. F. Stress generated in polyimide by excimer-laser irradiation. J. Appl. Phys. 74, 4181-4189 (1993).

22. Doukas, A. G. \& Flotte, T. J. Physical characteristics and biological effects of laserinduced stress waves. Ultrasound in Med. \& Biol. 22, 151-164 (1996).

23. Doukas, A. G., McAuliffe, D. J., Lee, S., Venugopalan, V. \& Flotte, T. J. Physical factors involved in stress-wave-induced cell injury: the effect of stress gradient. Ultrasound in Med. \& Biol. 21, 961-967 (1995).

24. Douki, T. et al. Stress-wave-induced injury to retinal pigment epithelium cells in vitro. Laser Surg. Med. 19, 249-259 (1996).

25. Kodama, T., Hamblin, M. R. \& Doukas, A. G. Cytoplasmic molecular delivery with shock waves: importance of impulse. Biophys. J. 79, 1821-1832 (2000).

26. Doukas, A. G. \& Kollias, N. Transdermal drug delivery with a pressure wave. $A d v$. Drug. Deliver Rev. 56, 559-579 (2004).

27. Chakravarty, P., Qian, W., El-Sayed, M. A. \& Prausnitz, M. R. Delivery of molecules into cells using carbon nanoparticles activated by femtosecond laser pulses. Nature Nanotechnology 5, 607-611 (2010).

28. Goldenstedt, C. et al. Delivery by shock waves of active principle embedded in gelatin-based capsules. Ultrason. Sonochem. 15, 808-814 (2008).

29. Wu, Junru. \& Nybordg, W. L. Ultrasound, cavitation bubbles and their interaction with cells. Adv. Drug Deliver Rev. 60, 1103-1116 (2008).

30. Bekeredjian, R. et al. Impact of microbubbles on shock wave-mediated DNA uptake in cells in vitro. Ultrasound in Med. \& Biol. 33, 743-750 (2007).

31. Coussious, C. C., Frany, C. H., Ter Harr, G. \& Roy, R. A. Role of acoustic cavitation in the delivery and monitoring of cancer treatment by high-intensity focused ultrasound (HIFU). Int. J. Hyperther. 23, 105-120 (2008).

32. Coussious, C. C. \& Roy, R. A. Applications of acoustics and cavitation to noninvasive drug delivery. Annu. Rev. Fluid Mech. 40, 395-420 (2008).

33. Johns, L. D. Nonthermal effects of therapeutic ultrasound: the frequency resonance hypothesis. J. Athl. Train. 37, 239-299 (2003).

34. Baker, K. G. A review of therapeutic ultrasound: biophysical effects. Phys. Ther. 81, 1351-1358 (2001).

35. Harris, G. R., Preston, R. C. \& DeReggi, A. S. The impact of piezoelectric PVDF on medical ultrasound exposure measurements, standards, and regulations. IEEE T. Ultrason. Ferr. 47, 1321-1335 (2000).

36. Ohtani, K., Numata, D., Takayama, K., Kobayashi, T. \& Okatsu, K. Experimental study of underwater rock drilling using a pulsed Ho:YAG laser-induced jets. Shock Waves 19, 403-412 (2009).

37. Strand, O. T., Goosman, D. R., Martinez, C., Whitworth, T. L. \& Kuhlow, W. W Compact system for high-speed velocimetry using heterodyne techniques. Rev. Sci. Instrum. 77, 083108 (2006)

38. Ponten, J. \& Macintyre, E. H. Long term culture of normal and neoplastic human glia. Acta Pathol. Microbiol. 74, 465-486 (1968).

39. Kahlert, U. D., Nikkhah, G. \& Maciaczyk, J. Epithelial-to-mesenchymal(-like) transition as a relevant molecular event in malignant gliomas. Cancer Lett. 331, 131-138 (2013)

40. Clark, M. J. et al. U87MG decoded: the genomic sequence of a cytogenetically aberrant human cancer cell line. PLoS genetics 6, e1000832 (2010).

41. Mitra, E. S. et al. Preclinical efficacy of the anti-hepatocyte growth factor antibody ficlatuzumab in a mouse brain orthotopic glioma model evaluated by bioluminescence, PET, and MRI. Clin. Cancer Res. 19, 5711-2721 (2013).

42. Wang, X. R. et al. Overexpressed let-7a inhibits glioma cell malignancy by directly targeting K-ras, independently of PTEN. Neuro. Ocol. 15, 1491-1501 (2013).

43. Saidi, A. et al. Experimental anti-angiogenesis causes upregulation of genes associated with poor survival in glioblastoma. Int. J. Cancer 15, 2187-2198 (2008).

44. Platte, M. A. Polyvinylidene fluoride needle hydrophone for ultrasonic applications. Ultrasonics 23, 113-118 (1985).

45. Juhasz, T., Hu, X. H., Turi, L. \& Bor, Z. Dynamics of shock waves and cavitation bubbles generated by picosecond laser pulses in corneal tissue and water. Laser Surg. Med. 15, 91-98 (1994).

46. Juhasz, T., Kastis, G. A., Suarez, C., Bor, Z. \& Bron, W. E. Time-resolved observations of shock waves and cavitation bubbles generated by femtosecond laser pulses in corneal tissue and water. Laser Surg. Med. 19, 23-31 (1996).

47. Vogel, A., Busch, S. \& Parlitz, U. Shock wave emission and cavitation bubble generation by picosecond and nanosecond optical breakdown in water. J. Acoust. Soc. Am. 100, 148-165 (1996).

48. Zel'Dovich, Y. B. \& Raizer, Y. P. Physics of Shock Waves and High-Temperature Hydrodynamic Phenomena. (Dover Publications, 2002).

49. Deblois, R. W. \& Bean, C. P. Counting and sizing of submicron particles by resistive pulse technique. Rev. Sci. Instrum. 41, 909-916 (1970).

50. Ohl, C. D. et al. Sonoporation from jetting cavitation bubbles. Biophys. J. 91, 4285-4295 (2006).

51. Ohl, C. D. \& Wolfrum, B. Detachment and sonoporation of adherent hela-cells by shock wave-induced cavitation. Biochim. Biophys. Acta 1624, 131-138 (2003).

52. Parsons, J. E., Cain, C. A., Abrams, G. D. \& Fowlkes, J. B. Pulsed cavitational ultrasound therapy for controlled tissue homogenization. Ultrasound in Med. \& Biol. 32, 115-129 (2006).

53. Brummer, F., Brauner, T. \& Hulser, D. F. Biological Effects of Shock-Waves. World J. Urol. 8, 224-232 (1990).

54. Delius, M. Medical applications and bioeffects of extracorporeal shock waves Shock Waves 4, 55-72 (1994). 
55. Steinhauser, M. O. Computational Multiscale Modeling and Simulation of Fluids and Solids: Theory and Applications. (Springer, Berlin, Heidelberg, New York, 2008).

56. Steinhauser, M. O. Computer Simulation in Physics and Engineering, (deGryuter, Leipzig, 2012).

57. Plimpton, S. Fast parallel algorithms for short-range molecular dynamics. J. Comp. Phys. 117, 1-19 (1995).

58. Ganzenmüller, G. C. \& Steinhauser, M. O. Shock-wave induced damage in lipid bilayers: a dissipative particle dynamics simulation study. Soft Matter 7 , 4307-4317 (2011)

59. Ganzenmüller, G. C. \& Steinhauser, M. O. Consistent temperature coupling with thermal fluctuations of smooth particle hydrodynamics and molecular dynamics. PLoS ONE 7, e51989 (2012).

60. Avalos, J. B. \& Mackie, A. D. Dissipative particle dynamics with energy conservation. Europhys. Lett. 40, 141-146 (1997).

61. Español, P. Dissipative particle dynamics with energy conservation. Europhys. Lett. 40, 631-636 (1997).

62. Steinhauser, M. O. Introduction to Molecular Dynamics Simulations: Applications in Hard and Soft Condensed Matter Physics, in: Wang, L. (editor) Molecular Dynamics - Studies of Synthetic and Biological Macromolecules. (InTech Open Science, 2012).

63. Steinhauser, M. O. Modeling dynamic failure behavior in granular and biological materials: Emerging new applications. AES Technical Reviews, Part C, IJATEMA $1,15-29$ (2012).

\section{Acknowledgments}

This work was financially supported by the Fraunhofer-Gesellschaft, e. V., Germany (grant No. 600016, Vintage Class Program: Shock-Wave Induced Destruction of Tumor Cells) and (grant No. 400017, Extracorporeal, Focused Ultrasound Therapy: Effectiveness, Simulation, and Planning of New Therapies). U. Kahlert acknowledges current support by the Dr. Mildred-Scheel stipend from the Deutsche Krebshilfe. We thank Dr. Ganzenmüller for generating some in-house simulation data. M.O. Steinhauser is grateful to Prof. Liangch Zhang (USNW Sydney, Australia), Prof. C. Coussious (Oxford University, UK) and Prof.
J.A. Käs (Universität Leipzig, Germany) for helpful discussions concerning cell culturing, tumor therapies, tumor cell destruction and MRI-guided HIFU treatments. M. Schmidt and M.O. Steinhauser thank Dr. S. Schiller (FRIAS, University of Freiburg) for helpful discussions.

\section{Author contributions}

M.S., U.K., J.O. and M.O.S. wrote the main manuscript text. U.K., J.W., D.M. and M.S cultured and prepared the tumor cells. U.K., J.M. and J.W. gave valuable comments on cell culturing and the biological interpretation and relevance of results. U.K. contributed the supplemental confocal microscopy images of U87 cells. M.S. prepared the supplemental figures. M.S. and M.O.S. conducted the laser experiments with tumor cells. M.S. analyzed the results of the laser experiments and prepared the corresponding figures. J.O. and B.M conducted the PDV experiments. B.M. wrote the software to analyze the raw data resulting from the PDV experiments. G.N. provided valuable comments to cell culturing and the sensitivity of U87 tumor cells to external stresses. The cell culturing was carried out in his cell laboratory. M.O.S. supervised the overall research work, contributed to the analysis and interpretation of experimental and computational results and edited the manuscript. The PDV and laser shock experiments were performed in his laser laboratory. The in-hose simulation data were produced under his supervision and guidance. All authors reviewed the final manuscript text.

\section{Additional information}

Supplementary information accompanies this paper at http://www.nature.com/ scientificreports

Competing financial interests: The authors declare no competing financial interests.

How to cite this article: Schmidt, M. et al. Characterization of a Setup to test the Impact of High-Amplitude Pressure Waves on Living Cells. Sci. Rep. 4, 3849; DOI:10.1038/srep03849 (2014)

(c) (i) () $\Theta$ This work is licensed under a Creative Commons Attribution-

NonCommercial-NoDerivs 3.0 Unported license. To view a copy of this license, visit http://creativecommons.org/licenses/by-nc-nd/3.0 